\title{
Imaging Characteristics of Dural Arteriovenous Fistulas Involving the Vein of Galen: A Comprehensive Review
}

Mohammad W. Kassem ${ }^{1}$, Paul J. Choi ${ }^{2}$, Joe Iwanaga ${ }^{3}$, Martin M. Mortazavi ${ }^{4}$, R. Shane Tubbs 5

1. Neuroscience Institute, Mercy Health, Toledo, USA 2. Surgery, Seattle Science Foundation, Seattle, USA 3. Medical Education and Simulation, Seattle Science Foundation, Seattle, USA 4. California Institute of Neuroscience, Los Robles Hospital and Medical Center 5. Neurosurgery, Seattle Science Foundation, Seattle, USA

$\square$ Corresponding author: Paul J. Choi, paulchoi92@gmail.com

Disclosures can be found in Additional Information at the end of the article

\section{Abstract}

Vein of Galen aneurysmal malformation (VGAM) is a rare angiopathy, which most commonly presents in infancy. Although very rare, it is associated with high morbidity and mortality rates. In order to minimize such morbid rates, a prompt diagnosis followed by a timely initiation of management is crucial. Multiple antenatal and postnatal imaging techniques for the diagnosis have been described and discussed in the literature. However, to our knowledge, a comprehensive review exploring such a list of imaging options for VGAM has never been established. We aim to review the diagnostic tools to aid in better understanding of the investigative modalities physicians may choose from when treating patients with a VGAM.

Categories: Pediatrics, Radiology, Neurosurgery

Keywords: vein of galen, aneurysmal malformation, arteriovenous fistula, imaging, diagnosis, prenatal

\section{Introduction And Background}

Dural arteriovenous fistula (DAVF) is a rare condition that arises from acquired arteriovenous shunts within the dura mater [1-3]. This cerebral arteriovenous malformation involves a single or multiple arterial vessel(s) that feed into a developing choroid, an accumulation of dysplastic vessels, and histologically appears as a congregation of veins, which are arterialized due to the high-pressure arterial system feeding into them. Classically, the choroid can either be fed via a single or by multiple vessel(s) [1,4]. DAVFs represent $10 \%$ to $15 \%$ of all cerebrovascular malformations. A rare subtype of a DAVF is the vein of Galen aneurysmal malformation

Received 02/06/2018 Review began 02/08/2018 Review ended 02/09/2018 Published 02/11/2018

\section{(c) Copyright 2018}

Kassem et al. This is an open access article distributed under the terms of the Creative Commons Attribution License CC-BY 3.0., which permits unrestricted use, distribution, and reproduction in any medium, provided the original author and source are credited.
(VGAM), which is also known as the median prosencephalic arteriovenous fistula (Figure 1) [1, 5]. This uncommon subtype of DAVF presents in a more serious and dramatic manner in infancy $[1,6]$.
How to cite this article

Kassem M W, Choi P J, Iwanaga J, et al. (February 11, 2018) Imaging Characteristics of Dural Arteriovenous Fistulas Involving the Vein of Galen: A Comprehensive Review. Cureus 10(2): e2180. DOI 10.7759 /cureus. 2180 


\section{Cureus}

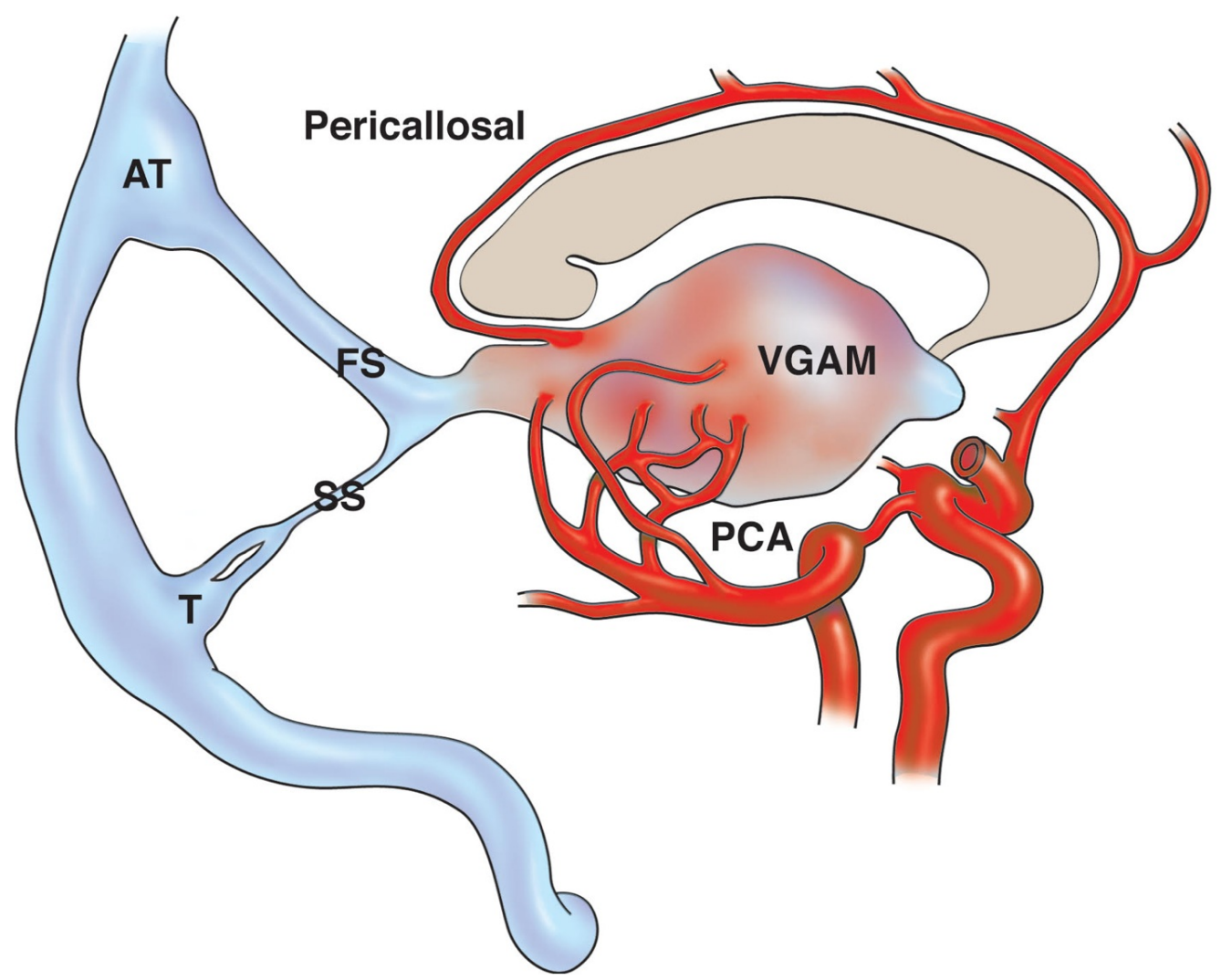

\section{FIGURE 1: Schematic drawing of the pathomechanism of a vein of Galen aneurysmal malformation}

Note the pericallosal artery and choroidal branches of the posterior cerebral artery (PCA) feeding the vein of Galen aneurysmal malformation (VGAM). Also note the prominent falcine sinus (FS) and the "accessory torcular" (AT).

SS: straight sinus; T: torcular.

VGAMs are more common in males, although Darji et al. claim that they affect boys and girls equally [6]. They make up less than $1 \%$ of all intracranial arteriovenous malformations (AVMs) but are $30 \%$ of all pediatric intracranial AVMs [7-9]. VGAMs are also the most commonly diagnosed antenatal intracranial AVMs [7]. Their most common presenting symptoms include mild pulsatile tinnitus, hydrocephalus secondary to venous hypertension or aqueduct stenosis, developmental delays, focal neurological deficits, and an intracranial hemorrhage (ICH) [2, 5, 67]. Other presentations include epistaxis, proptosis, and less commonly, visual loss from bilateral optic disc atrophy [4].

A VGAM (as seen in Figure 2) that presents in the first trimester (6-11 week) of fetal life is of great concern due to its propensity to cause high-output cardiac failure, the result of an increased venous return and subsequent contractility, cardiac output, and eventual high demand for oxygen $[4,6-8,10]$. The patients may have cardiomegaly and pulmonary hypertension, which may lead to fetal hydrops $[4,8,11]$. The VGAM is also known as a rare subtype of falcotentorial DAVF, which is a well-documented cause of an ICH [7, 12]. Identifying VGAM early is crucial in preventing developmental delays, intellectual disabilities, seizures, and a spontaneous ICH $[4-5,7]$. 


\section{Cureus}

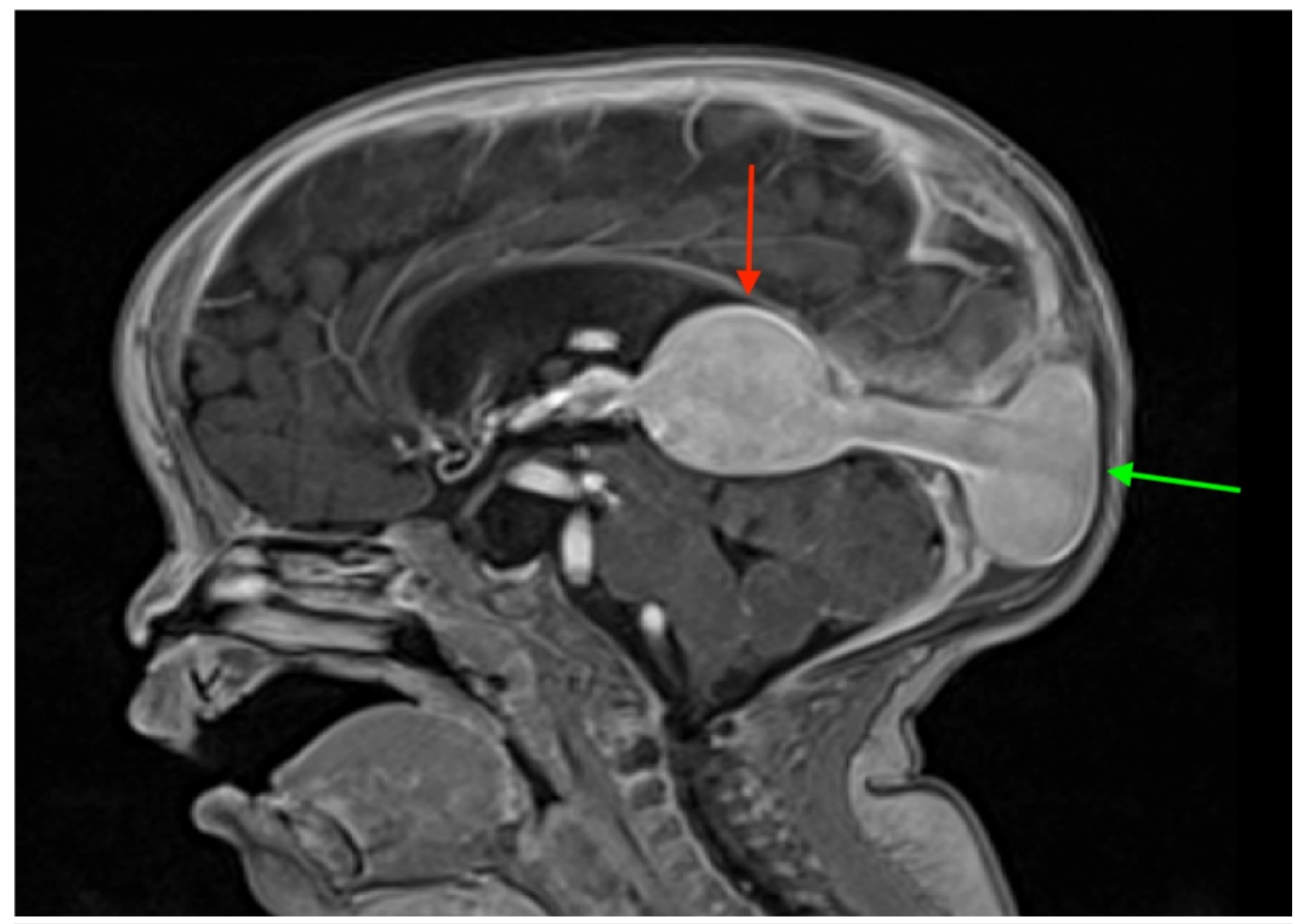

FIGURE 2: Sagittal T1-weighted MRI of a child with a VGAM (red arrow)

Also note the enlarged torcular herophili (TH) (green arrow).

MRI: magnetic resonance imaging; VGAM: vein of Galen aneurysmal malformation.

The mortality rate of this condition is as high as $15 \%$ if identified timely and managed promptly with endovascular embolization, which is the current treatment of choice for it yields the most favorable outcome $[4-5,9,13]$. However, if such an early detection and an expedited management are not established, a VGAM bestows a dismal 76.7\% mortality rate if untreated, and the patients who are treated with a microsurgery take in a mortality rate of $39.4 \%$ (Figure 3 ) $[6,13]$. Even if the patient survives, there is a $30 \%$ risk of developing a neurological complication $[3,9,11]$. 


\section{Cureus}

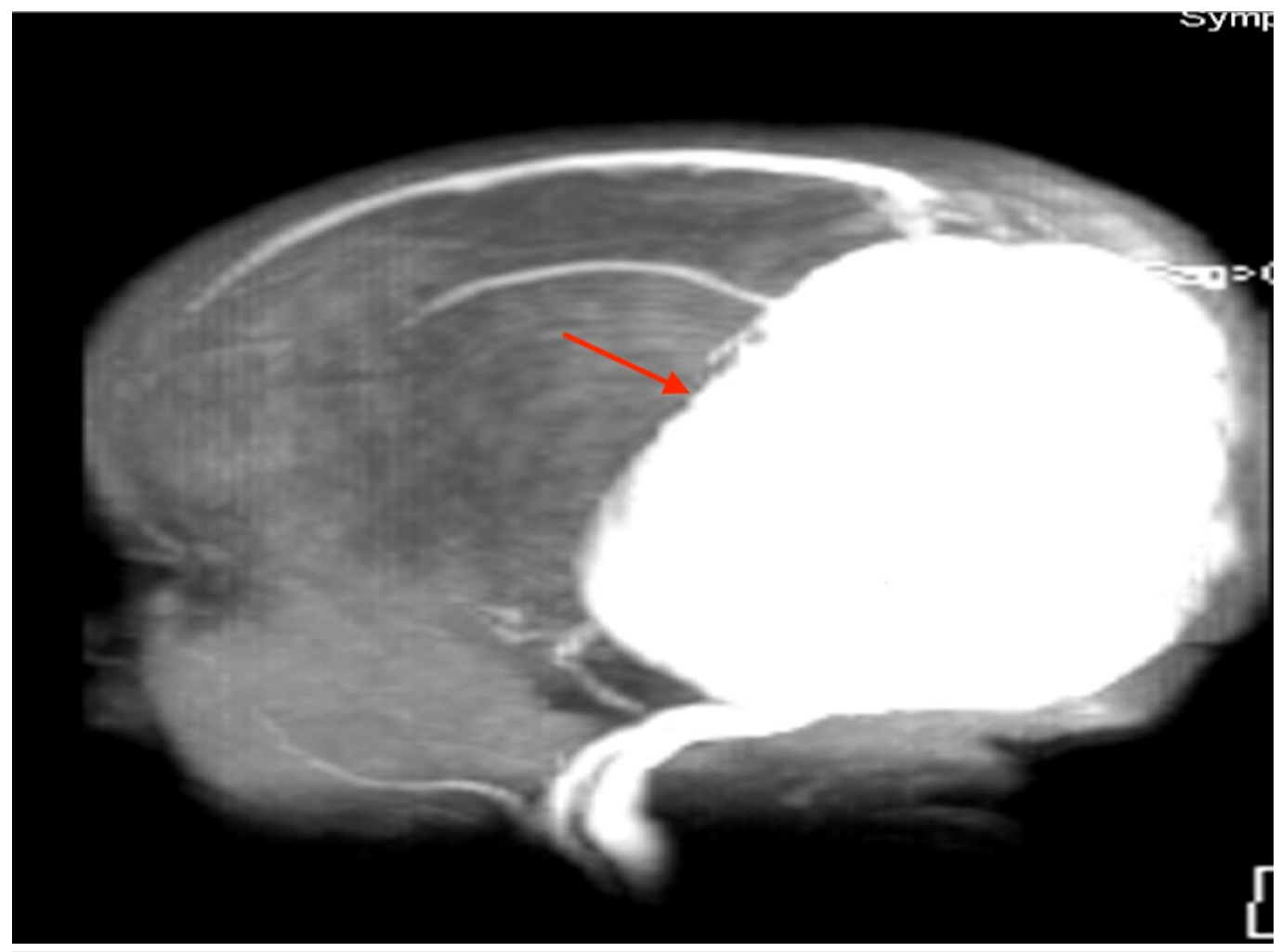

FIGURE 3: MRI scout image noting the large VGAM (red arrow) seen in the patient shown in Figure 2

MRI: magnetic resonance imaging; VGAM: vein of Galen aneurysmal malformation.

\section{Review}

\section{Imaging modalities for the diagnosis of VGAM}

Imaging is a critical aspect in the diagnosis of a VGAM. A VGAM requires a sensitive imaging input for an early detection, which would allow for a careful obstetric monitoring in order to prevent a fatal ICH $[1,5,11-12,14]$. A prenatal diagnosis of a VGAM offers an opportunity to plan the delivery at a center equipped with a multidisciplinary team who can provide the ideal care [11]. This provides an additional benefit of allowing the parents to prepare, emotionally, for a palliative intervention and avoid unnecessary non-surgical and surgical interventions [15]. Diagnosing a VGAM prenatally can make a significant impact in managing the patient postnatally.

\section{Ultrasound}

A two-dimensional real-time ultrasonography can identify an aneurysm as a hypoechoic midline mass posterior to the roof of the third ventricle [2, 6, 8, 15-17]. Using color Doppler technology as an adjunct further demonstrates the hemodynamics, i.e., increased middle cerebral artery (MCA) pressure, high turbulence, and bidirectional blood flow [15], within the aneurysm and aids in ruling out other midline lesions, such as pineal region meningiomas over the vein of Galen [17-19], that appear as a midline solid structure on initial ultrasound [3, 6, 16]. The ultrasonography can also help physicians in identifying other associated anomalies, such as hydrocephalus, ventriculomegaly, and left ventricular dysfunction [5-6, 16-17]. The use of ultrasonography is also of importance in postnatal follow-up on an endovascularly treated VGAM to monitor its potential sequelae [16]. Gun et al. recommend utilization of three- 


\section{Cureus}

dimensional ultrasonography for antenatal diagnosis of a VGAM for its accuracy in measuring the volume and shape of the lesion [8, 11, 20].

\section{Computed tomography (CT)}

Postnatal contrast-enhanced axial CT of the brain can show the detailed multilobulated morphology of the aneurysmal sac, as well as the dilatation of the ventricular system [2-3, 21]. The CT also shows the hypodense periventricular white matter and diffuse cerebral atrophy associated with a VGAM [21]. Cases of spontaneous thrombosis of VGAMs, in which the thrombus sits within the aneurysmal sac, have been reported previously on multiple occasions $[2,21]$. Such a thrombus would show up as a heterogeneous mass of hyperdensity, hypodensity, and isodensity, and appears as the "target sign" (peripheral blood flow around the thrombus) under contrast-enhanced CT [21]. Further, the CT technology can highlight dilated collateral veins and hydrocephalus [6, 21].

\section{Magnetic resonance imaging (MRI)}

MRI is becoming the imaging modality of choice for VGAM because of its non-invasive nature and its ability to delineate soft tissues effectively i.e., accurately visualizes hydrocephalus, cortical atrophy and injury, and cardiac complications, and to differentiate a VGAM from a cerebral AVM that drains into the vein of Galen (Figures 4-6) [2, 8, 11, 22-23]. Because of its ability to show subtle contrast changes, MRI is ideal for visualizing changes within the cerebral parenchyma and capturing a detailed picture of the ventricular system [5, 22]. MRI is capable of identifying the specific location of a nidus, fistula, venous sac, and the venous vessels [22]. MRI elucidates the major feeding arterial branches more clearly than the traditional CT method [24]. For instance, Saliou et al. reported a fetal MRI brain scan's accuracy in predicting cardiopulmonary failure at birth and encephalopathic sequelae of VGAM by capturing the M2 and M3 branches of the MCA feeding into the malformation [25].

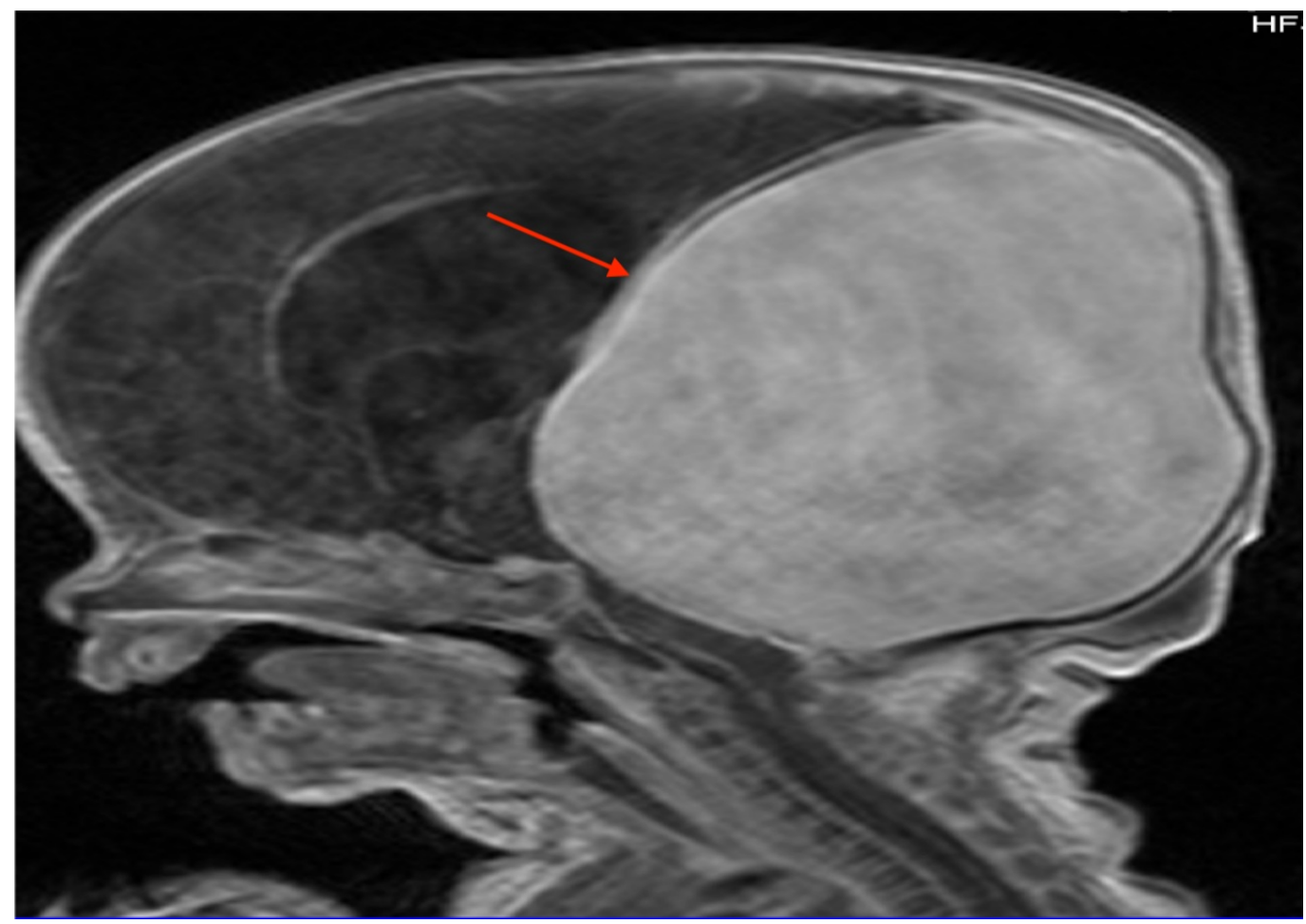

FIGURE 4: Post-contrasted MRI of a patient with a large VGAM (red arrow) seen in Figures 2 and 3 


\section{Cureus}

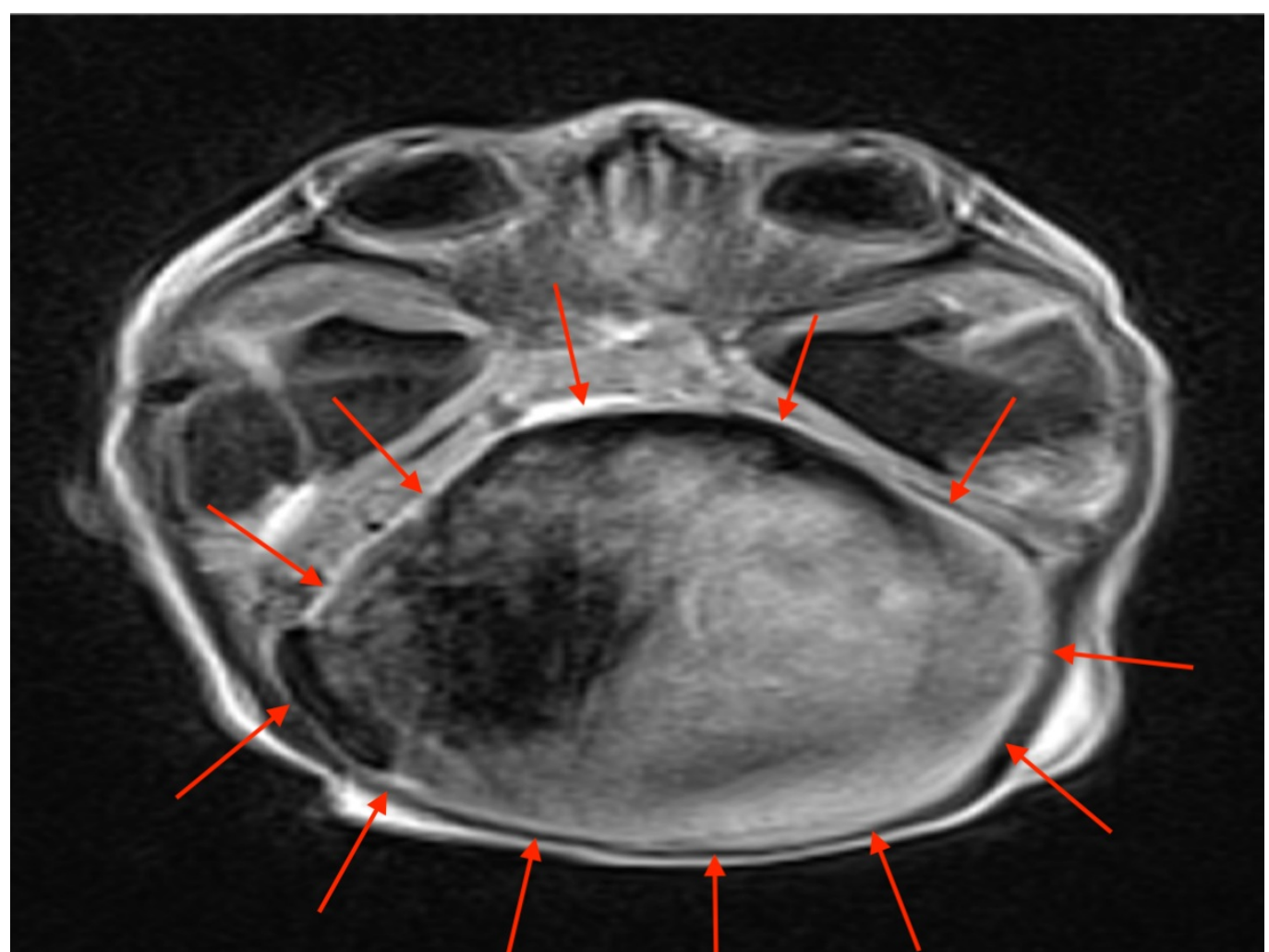

FIGURE 5: Axial T1-weighted MRI of the patient above with a large VGAM (red arrows)

MRI: magnetic resonance imaging; VGAM: vein of Galen aneurysmal malformation. 


\section{Cureus}

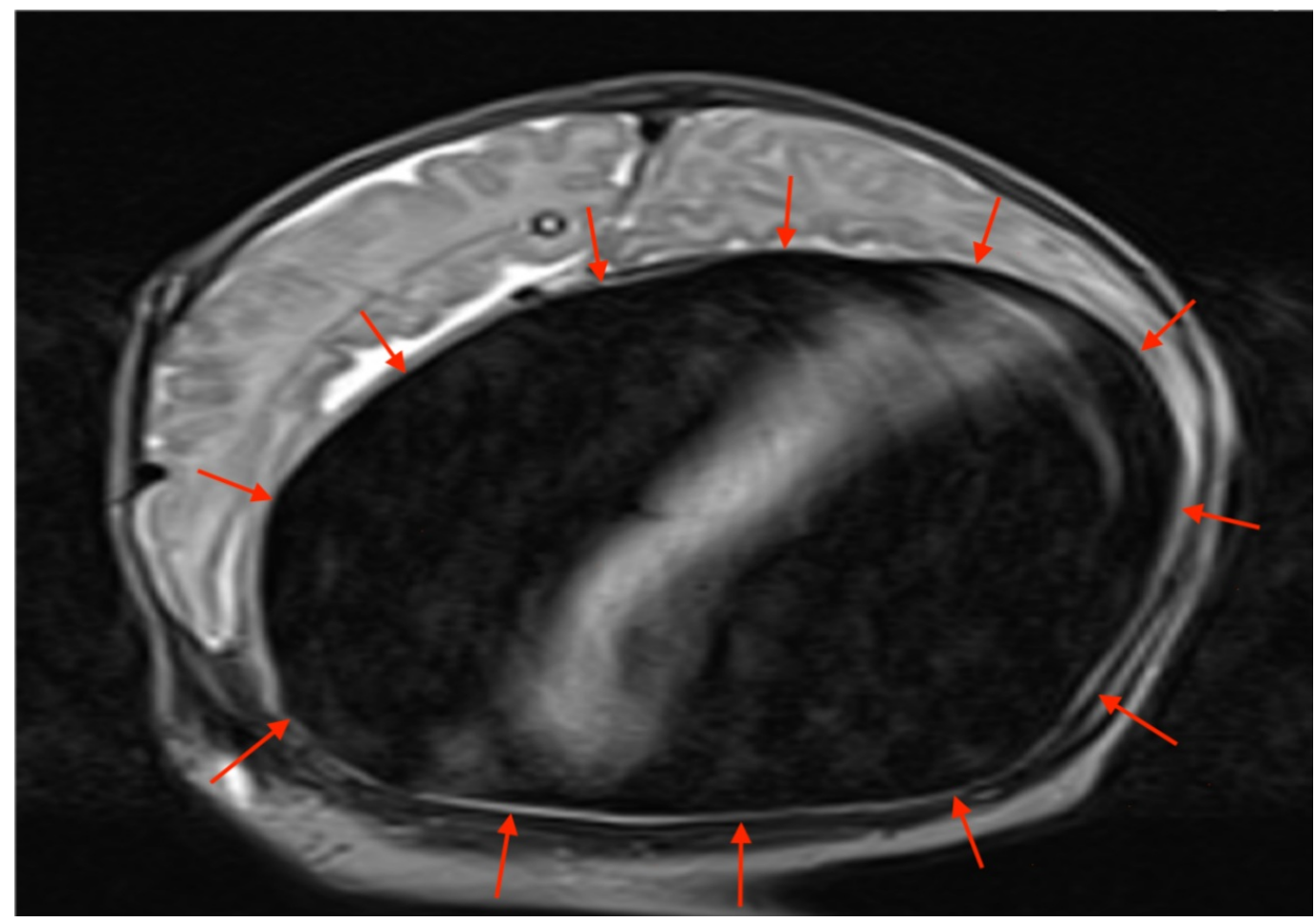

\section{FIGURE 6: Coronal T2-weighted MRI of the patient above noting the large VGAM (red arrows)}

MRI: magnetic resonance imaging; VGAM: vein of Galen aneurysmal malformation.

MRI is also useful in excluding other pathologic lesions such as a choroid plexus cyst and pineal tumor and pinpointing parenchymal injury, which requires an aggressive intervention $[8,15$, 17]. In addition, the magnetic resonance angiography (MRA) is a noninvasive alternative imagining choice for the initial evaluation of a suspected VGAM [24]. It has recently been established that fetal MRI is superior to color Doppler in the diagnosis of a VGAM prenatally [8, 23, 26]. Moreover, MRI and MRA have a critical role in understanding the lesion prior and at the time of endovascular embolization respectively [11].

\section{Digital subtraction angiography/fluoroscopy (DSA)}

DSA is the gold standard in the postnatal diagnosis of a VGAM (Figure 7) [27]. DSA allows for easier visualization of small arteries, which feed into the fistula and it also shows the relationship between the arteriovenous shunt and the venous drainage [6, 27]. The traditional $\mathrm{X}$-ray image of the cranium may aid in the identification of such a condition; it may identify calcification within the wall of the aneurysmal sac and plain radiography of the chest helps rule out congestive heart failure caused by the VGAM (Figure 8) [16, 28]. 


\section{Cureus}

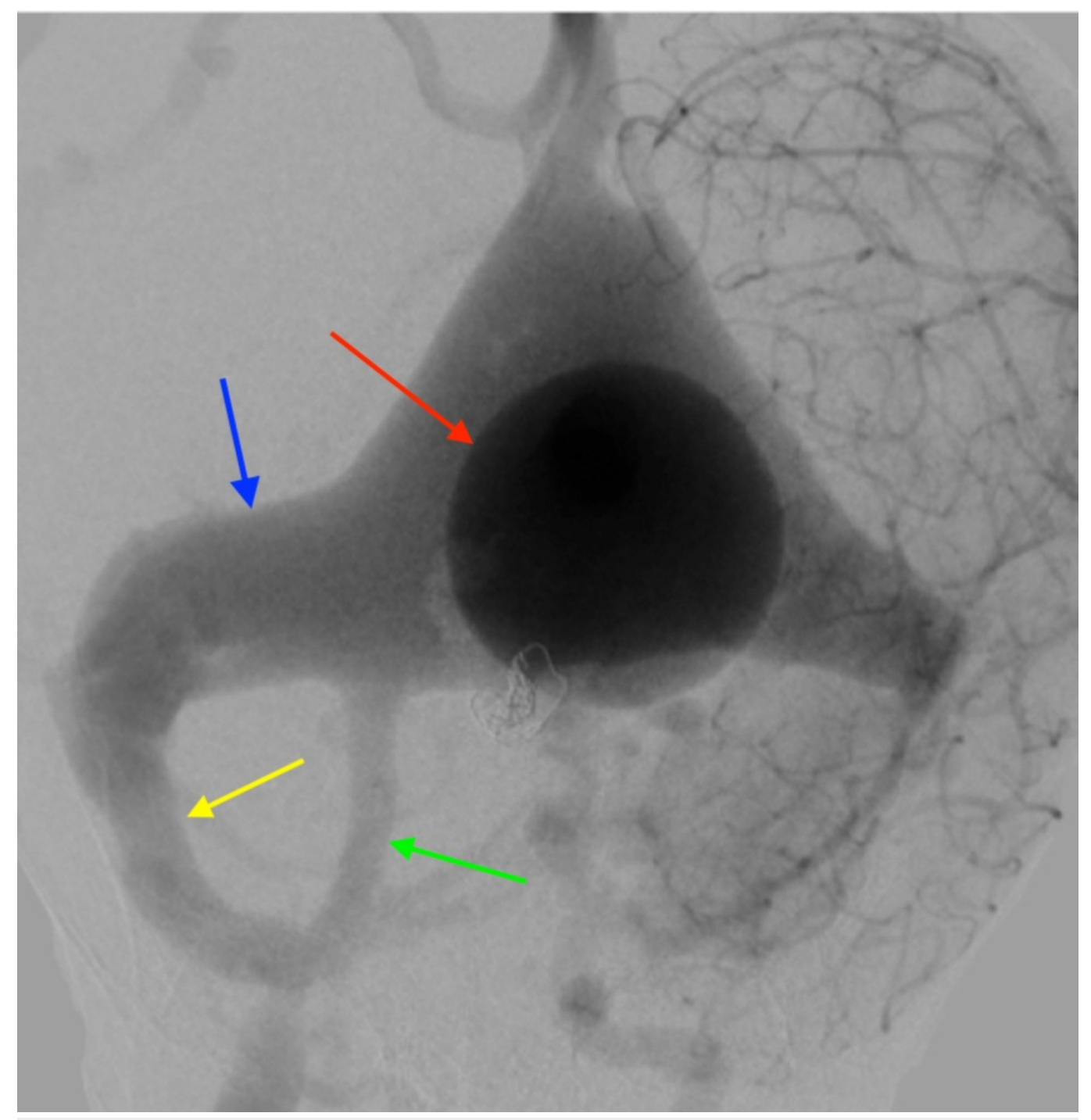

FIGURE 7: Angiogram, posterior view, noting the VGAM (red arrow), dilated TS (blue arrow), OOS variant (green arrow), and SS (yellow arrow)

VGAM: vein of Galen aneurysmal malformation; TS: transverse sinus; OOS: oblique occipital sinus; SS: sigmoid sinus. 


\section{Cureus}

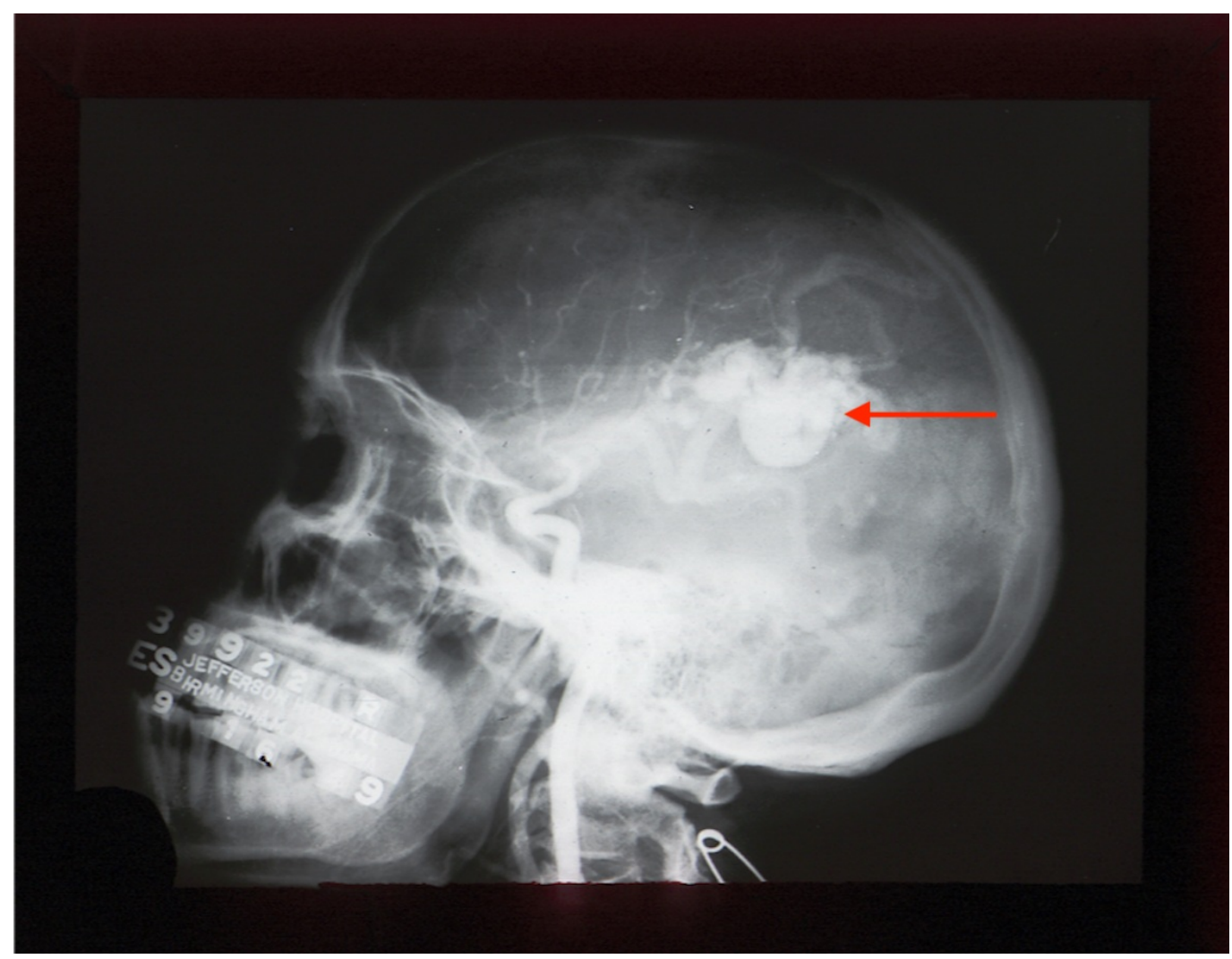

FIGURE 8: Lateral skull radiograph noting a VGAM (red arrow)

VGAM - vein of Galen aneurysmal malformation

\section{Conclusions}

VGAM is a rare condition found mainly in the pediatric population and carries a dismal prognosis if undealt promptly with an appropriate imaging study followed by an immediate initiation of an endovascular intervention. Prenatal diagnosis would not only allow time for the transfer of the pregnant mother to a well-equipped medical center for the delivery and care of a neonate with a VGAM, but it may also provide more time for the parents to emotionally prepare for a palliative care and help avoid unnecessary costly interventions. For such reasons, in utero diagnosis is becoming more common with improved fetal imaging technology i.e., threedimensional ultrasonography and MRI of the fetal brain, which can predict prognosis. An early postnatal diagnosis is also crucial in achieving a better outcome. The appropriate use of advanced imagining techniques allows for an earlier intervention, ultimately leading to improved quality of the children's remaining decades of life.

\section{Additional Information}

\section{Disclosures}

Conflicts of interest: In compliance with the ICMJE uniform disclosure form, all authors declare the following: Payment/services info: All authors have declared that no financial support was received from any organization for the submitted work. Financial relationships: All authors have declared that they have no financial relationships at present or within the previous three years with any organizations that might have an interest in the submitted work. Other relationships: All authors have declared that there are no other relationships or activities that could appear to have influenced the submitted work. 


\section{References}

1. Huded V, Moeed SZ, Desouza R, Tripathi R, Nair R: Endovascular treatment of vein of Galen dural arteriovenous fistula presenting as dementia. Ann Indian Acad Neurol. 2014, 17:451454. 10.4103/0972-2327.144037

2. Kebede T, Hawaz Y, Assefa G: CT and color Doppler diagnosis of the vein of Galen malformations with hydrocephalus: a case series report of rare intracranial vascular malformations. Ethiop Med J. 2013, 51:77-83.

3. Lopez-Cepero R, de la Vega A, Lynch L: Prenatal diagnosis of a vein of Galen aneurysmal malformation using color Doppler ultrasound: a case report. Bol Asoc Med P R. 2013, 105:3235.

4. Agarwal H, Sebastian LJ, Gaikwad SB, Garg A, Mishra NK: Vein of Galen aneurysmal malformation-clinical and angiographic spectrum with management perspective: an institutional experience. J Neurointerv Surg. 2017, 9:159-164. 10.1136/neurintsurg-2015012137

5. George Zaki Ghali M: Galenic pial arteriovenous fistulas: Angioarchitecture, clinical presentation, and therapeutic considerations. Clin Anat. 2017, 10.1002/ca.23004

6. Darji PJ, Gandhi VS, Banker H, Chaudhari H: Antenatal diagnosis of aneurysmal malformation of the vein of Galen. BMJ Case Rep. 2015, 10.1136/bcr-2015-213785

7. Chow ML, Cooke DL, Fullerton HJ, et al.: Radiological and clinical features of vein of Galen malformations. J Neurointerv Surg. 2015, 7:443-448. 10.1136/neurintsurg-2013-011005

8. Herghelegiu D, Ionescu CA, Pacu I, Bohiltea R, Herghelegiu C, Vladareanu S: Antenatal diagnosis and prognostic factors of aneurysmal malformation of the vein of Galen: a case report and literature review. Medicine. 2017, 96:e7483. 10.1097/MD.0000000000007483

9. Heuer GG, Gabel B, Beslow LA, et al.: Diagnosis and treatment of vein of Galen aneurysmal malformations. Childs Nerv Syst. 2010, 26:879-887. 10.1007/s00381-009-1063-8

10. Barajas-Gamboa JS, Diaz-Perez JA, Leon-Camargo Y, Gonzalez-Gomez CA, Sandoval-Gomez C: Systolic heart murmur as first manifestation of high output heart failure due to the vein of galen malformation. Arch Cardiol Mex. 2012, 82:214-217. 10.1016/j.acmx.2012.04.002

11. Karadeniz L, Coban A, Sencer S, Has R, Ince Z, Can G: Vein of Galen aneurysmal malformation: prenatal diagnosis and early endovascular management. J Chin Med Assoc. 2011, 74:134-137. 10.1016/j.jcma.2011.01.029

12. Sahlein DH, Mora P, Becske T, et al.: Features predictive of brain arteriovenous malformation hemorrhage: extrapolation to a physiologic model. Stroke. 2014, 45:1964-1970.

10.1161/strokeaha.114.005170

13. Khullar D, Andeejani AM, Bulsara KR: Evolution of treatment options for vein of Galen malformations. J Neurosurg Pediatr. 2010, 6:444-451. 10.3171/2010.8.peds10231

14. Fok EW, Poon WL, Tse KS, et al.: Angiographic factors associated with haemorrhagic presentation of brain arteriovenous malformation in a Chinese paediatric population. Hong Kong Med J. 2015, 21:401-406. 10.12809/hkmj144339

15. Nuutila M, Saisto T: Prenatal diagnosis of vein of Galen malformation: a multidisciplinary challenge. Am J Perinatol. 2008, 25:225-227. 10.1055/s-2008-1066877

16. Campi A, Scotti G, Filippi M, Gerevini S, Strigimi F, Lasjaunias P: Antenatal diagnosis of vein of Galen aneurysmal malformation: MR study of fetal brain and postnatal follow-up. Neuroradiology. 1996, 38:87-90. https://doi.org/10.1007/BF00593231

17. Michaels AY, Sood S, Frates MC: Vein of Galen aneurysmal malformation. Ultrasound Q. 2016, 32:366-369. 10.1097/ruq.0000000000000222

18. Gokhale S, Laskowitz DT: Teaching neuroimages: vein of Galen aneurysm mimicking pineal mass in a young adult. Neurology. 2013, 80:240. 10.1212/WNL.0b013e318294b3df

19. Inoue A, Ohnishi T, Kohno S, et al.: Two cases of pineal-region meningiomas derived from arachnoid membrane over the vein of Galen without dural attachment. World J Surg Oncol. 2015, 13:226. 10.1186/s12957-015-0645-Z

20. Gun I, Mungen E, Kurdoglu M, Babacan A, Muhcu M, Atay V: Is prenatal volumetric evaluation of aneurysm of the vein of Galen important in the prediction of adverse fetal outcome?. J Clin Ultrasound. 2010, 38:443-445. 10.1002/jcu.20672

21. Nikas DC, Proctor MR, Scott RM: Spontaneous thrombosis of vein of Galen aneurysmal malformation. Pediatr Neurosurg. 1999, 31:33-39. 10.1159/000028828

22. Alvarez H, Garcia Monaco R, Rodesch G, Sachet M, Krings T, Lasjaunias P: Vein of galen 


\section{Cureus}

aneurysmal malformations. Neuroimaging Clin N Am. 2007, 17:189-206.

10.1016/j.nic.2007.02.005

23. Kalra V, Malhotra A: Fetal MR diagnosis of vein of Galen aneurysmal malformation. Pediatr Radiol. 2010, 40:155. 10.1007/s00247-010-1813-5

24. Sener RN: MR angiography of the vein of Galen malformation . Clin Imaging. 1996, 20:243246.

25. Saliou G, Vraka I, Teglas JP, et al.: Pseudofeeders on fetal magnetic resonance imaging predict outcome in vein of Galen malformations. Ann Neurol. 2017, 81:278-286. 10.1002/ana.24873

26. Wagner MW, Vaught AJ, Poretti A, Blakemore KJ, Huisman TA: Vein of galen aneurysmal malformation: prognostic markers depicted on fetal MRI. Neuroradiol J. 2015, 28:72-75. 10.15274/nrj-2014-10106

27. Gupta AK, Varma DR: Vein of Galen malformations: review. Neurol India. 2004, 52:43-53.

28. Sharma S, Sankhyan N, Kumar A: Teaching NeuroImages: Macrocephaly with subcortical calcifications in vein of Galen aneurysmal malformation. Neurology. 2009, 72:111. 10.1212/WNL.0b013e3181a71164 